\title{
Estimating the sea state bias of the TOPEX and POSEIDON altimeters from crossover differences
}

\author{
Philippe Gaspar, Françoise Ogor, Pierre-Yves Le Traon, and Ouan-Zan Zanife \\ Collecte Localisation Satellites, Space Oceanography Group, Toulouse, France
}

Abstract. TOPEX (Ku band) and POSEIDON altimeter measurements at crossover points are used to estimate the sea state bias (SSB) of these two instruments. Different $\mathrm{SSB}$ models are tested, ranging from a constant fraction of the significant wave height (SWH) to more elaborate models involving up to four adjustable parameters. For TOPEX, the data show a decrease in the magnitude of the relative bias (SSB/SWH) with SWH. This behavior is well reproduced using a simple empirical model with two adjustable parameters. The three-parameter SSB model used in the NASA geophysical data records does well in explaining the wind-induced variations of the bias. A model including four adjustable parameters is needed to account for both the wind- and SWHrelated variability of the SSB. POSEIDON data analysis reveals a significantly larger $\mathrm{SSB}$ than for TOPEX. This bias seems to consist of a skewness plus tracker bias of -2 to $-3 \%$ of SWH superimposed on a natural EM bias whose wind- and SWH-related variations are similar to those of TOPEX.

\section{Introduction}

TOPEX/POSEIDON is designed to be a high-precision satellite altimetric mission dedicated to the observation of the ocean general circulation. It takes advantage of new advances in altimeter technology and altimeter data processing to reduce all possible measurements errors. As the data show, the result is the most accurate satellite altimeter mission to date. In previous missions, the error in the radial position of the satellite dominated the altimetric error budget. The TOPEX/POSEIDON orbit error is now below $5 \mathrm{~cm}$ RMS [e.g., Noul et al., this issue]. Altimeter sea state bias is the second largest source of error in the TOPEX/ POSEIDON provisional error budget established by Stewart et al. [1986]. Its estimated uncertainty is about $2 \mathrm{~cm}$ RMS. The work reported in this paper is a contribution toward a better estimation of this bias.

Sea state bias (SSB) has three well-identified components: electromagnetic (EM) bias, skewness bias, and tracker bias. The EM bias is the difference between the mean height of the sea surface specular facets and the mean height of the sea level. Observations and theory show that the mean height of the specular facets is below mean sea level, the difference being proportional to the significant wave height (SWH). Typical values of EM bias are between -1 and $-4 \%$ of SWH. The skewness and tracker biases are due to inaccurate tracking of mean height of the specular facets by the altimeter tracker. As $F u[1990$, p. 25] explains: "The altimeter onboard tracker is actually designed to track the median height of the specular facets. The difference between the median and the mean of the height of the specular facets is called the skewness bias because it is directly related to the skewness of the distribution of the specular facets. The error in the tracker's determination of the median height is called the tracker bias." The skewness bias is generally smaller

Copyright 1994 by the American Geophysical Union.

Paper number 94JC01430.

$0148-(0227 / 94 / 94 \mathrm{JC}-01430 \$ 05.00$ than the EM bias while different tracking problems can result in a nonnegligible tracker bias.

Several papers concerning the sea state bias, or more specifically the EM bias, have been published, including a few theoretical works and many experimental studies. Jackson [1979] presented the first theoretical analysis of the EM bias. His work was based on the geometrical optics approximation to radar backscatter and assumed nonGaussian distribution of surface elevation and slopes as derived from the statistical theory of Longuet-Higgins [1963] for weakly nonlinear gravity waves. His approach was restricted to a unidirectional sea. Barrick and Lipa [1985] and Srokosz [1986] extended this work taking into account two-dimensional surface slopes. They showed that in this context the EM bias (EMB) can be expressed as

$$
\mathrm{EMB}=-\left(\lambda_{2} / 8\right) \mathrm{SWH}
$$

where $\lambda_{2}$ is a function of second-and third-order moments of the joint probability density function of the surface elevation and slopes. Unfortunately, (1) cannot be used directly for operational evaluation of the SSB as the statistical moments involved in the computation of $\lambda_{2}$ cannot be accurately estimated from present altimeter measurements [Rodriguez, 1988]. Jackson [1979] and Barrick and Lipa [1985] provided quantitative estimates of the EM bias for specific wave spectra only.

More recently, Rodriguez et al. [1992] investigated the physics of the EM bias conducting a set of numerical scattering experiments. This approach was chosen to minimize the number of assumptions both in the electromagnetic calculations (applicability of geometrical optics is not assumed) and in the characterization of the surface (for example, the surface is simulated without making assumptions about the high-order moments of the distribution of the surface elevation and slopes). In addition, the simulations of Rodriguez, et al. [1992] include the small gravity-capillary waves and allow them to be modulated by large waves. However, the computations are based on a specified wave 
spectrum that only approximates the true wave spectrum and is largely uncertain in its small-wave part. Furthermore, because of computational limitations, only one-dimensional simulations are performed. Accordingly, the authors do not claim to make exact predictions of the EM bias magnitude but rather insist on the behavior of the simulated bias as a function of the radar frequency and wind speed. Results concerning the wind speed dependence are specially interesting and will be discussed later in this paper.

This short review of theoretical works clearly shows the limitations of the present theories and their inability to provide simple means for accurately determining the EM bias from available altimeter measurements. In another approach, a number of research groups attempted direct determination of SSB either in the laboratory [e.g., Branger et al., 1993] or from aircraft or towers [e.g., Melville et al., 1991; Walsh et al., 1991; Hevizi et al., 1993]. They then tried to empirically relate the measured bias to easily accessible sea surface parameters. Such studies are relatively few and often limited to a narrow range of wind and sea state conditions. Still, they provide interesting information about the SSB variability as a function of different wind- or wave-related parameters.

Empirical studies have also been performed to extract from the altimeter data themselves the fraction of the signal that correlates with sea state-related parameters [e.g., Born et al., 1982; Ray and Koblinsky, 1991; Fu and Glazman, 1991]. The advantage is that huge data sets, covering a wide range of sea surface conditions, are available for experiments. The drawback is that the EM bias is only one part of the extracted signal. Other sea state related signals, including the skewness and tracker bias, are also extracted. Preliminary data processing is needed to eliminate or at least reduce such unwanted signals. Previous studies, mostly with Geosat data, were hampered by the presence of a relatively large orbit error which was not easily eliminated without affecting the SSB signal [Zlotnicki et al., 1989]. TOPEX/ POSEIDON provides us with a much better data set. Interesting results can thus reasonably be expected from new empirical studies of the SSB, like the one presented in this paper.

Our SSB estimation method is presented in the next section. Special attention is paid to data processing and noise reduction techniques. The data set is described in section 3. Estimates of the TOPEX $\mathrm{Ku}$ band SSB are obtained in section 4, using different types of models including the Fu and Glazman [1991] model and a whole family of empirical models. Both the global and regional performances of the models are analyzed. In section 5 the same models are used to estimate the POSEIDON SSB. The last section summarizes and discusses the main results of this study.

\section{Sea State Bias Estimation Technique}

Our problem is that of extracting a small signal, the sea state bias (SSB), from altimeter measurements containing much stronger signals and errors. In such conditions the signal to be extracted is best estimated when (1) a precise model of it is available and (2) when noise, or other signals, are reduced to a minimum before estimating the signal. Unfortunately, theoretically derived SSB models yield rather limited, often qualitative, results. As a consequence, most present models have empirical rather than theoretical bases. Such models might also describe, at least partly, non-SSB signals within the altimeter data. The estimated SSB can thus be polluted by other effects with similar functional behavior. In such circumstances it is specially important that most of the unwanted signals or noise be eliminated before estimating SSB. However, one must be extremely cautious as some noise reduction techniques can easily corrupt the weak SSB signal in the data. In general, we prefer a reliable estimate of SSB with large error bars to a less uncertain biased estimate.

\subsection{Content of Altimeter Data}

The basic data are altimeter-derived sea surface height (SSH) relative to the reference ellipsoid. It is obtained by forming the difference between the satellite altitude $(H)$ and the altimeter range measurement $\left(h_{a}\right)$. Here we cannot, a priori, correct the altimeter range for the sea state bias we are trying to estimate. We thus have to work with an uncorrected range

$$
h_{a}^{\prime}=h_{a}-\mathrm{SSB}
$$

and an uncorrected sea surface height ( $\left.\mathrm{SSH}^{\prime}\right)$ that contains the geoid signal $\left(h_{g}\right)$, dynamic topography $(\eta)$, and SSB:

$$
\mathrm{SSH}^{\prime}=H-h_{a}^{\prime}=h_{g}+\eta+\mathrm{SSB}
$$

Using the generic notation $x=x_{m}+\varepsilon_{x}$, where $x_{m}$ is the measured or estimated value of any variable $x$ and $\varepsilon_{x}$ its error, (3a) can be rewritten

$$
\mathrm{SSH}_{m}^{\prime}+\varepsilon_{\mathrm{SSH}^{\prime}}=H_{m}-h_{a m}^{\prime}+\varepsilon_{H}-\varepsilon_{h_{a}^{\prime}}=h_{g}+\eta+\mathrm{SSB}
$$

hence

$$
\mathrm{SSH}_{m}^{\prime}=\mathrm{SSB}+h_{g}+\eta-\varepsilon_{H}+\varepsilon_{h_{a}^{\prime}}
$$

where $\varepsilon_{H}$ is orbit error and $\varepsilon_{h_{a}^{\prime}}$ the altimetric measurement error including all instrumental and geophysical correction errors, except SSB. If one tries to retrieve the SSB directly from $\mathrm{SSH}_{m}^{\prime}$ data, (4) indicates that the noise affecting the estimation process will be the sum of the geoid signal, the dynamic topography and the orbit and altimetric measurement errors.

\subsection{Dealing With the Geoid and Dynamic Topography Signal}

The geoid is, by far, the largest signal in the data, of the order of meters to tens of meters. It is, fortunately, easily eliminated by forming differences between measurements taken at the same geographic locations, either along collinear tracks or at crossover points. Differences can be between individual measurements made at different times or between individual measurements and a mean. When forming such differences, (4) directly yields

$$
\Delta \mathrm{SSH}_{m}^{\prime}=\Delta \mathrm{SSB}+\Delta \eta-\Delta \varepsilon_{H}+\Delta \varepsilon_{h_{u}^{\prime}}
$$

where $\Delta$ denotes a difference. Measurement differencing actually eliminates any time-invariant component in the data: the geoid but also the steady part of the dynamic topography.

Most empirical studies on the SSB of Seasat or Geosat are based on collinear differences [Born et al., 1982; Douglas and Agreen, 1983; Zlotnicki et al., 1989; Fu and Glazman, 
1991; Ray and Koblinsky, 1991]. Forming differences between a mean track and individual passes is the most usual differencing technique. However, the combination of data gaps and orbit errors complicates the estimation of the mean [e.g., Chelton et al., 1990] and the nominal SSB correction used to compute the mean track can bias later SSB estimates [Ray and Koblinsky, 1991]. Accordingly, recent works on SSB [Fu and Glazman, 1991; Ray and Koblinsky, 1991] tend to use collinear differences between individual tracks.

Collinear differencing produces vast amounts of data. Along-track measurements are about $7 \mathrm{~km}$ apart (for 1-s averages). They are thus highly correlated as the typical oceanic correlation scales are of the order of several tens of kilometers, for the mesoscale, or larger. Subsampling of the data is thus perfectly appropriate for the purpose of SSB determination. Forming crossover differences is a very simple and efficient way to do so. In this study we will take advantage of a large TOPEX/POSEIDON crossover data set. For this satellite, the distance between adjacent crossover points is close to $150 \mathrm{~km}$ at midlatitudes. A specially interesting property of crossover points is that differences can be taken between data from the same repeat cycle. The time lag between differenced measurements is then relatively small. In our TOPEX/POSEIDON data set, its mean value is close to 3.5 days. The change in dynamic topography $\Delta \eta$ is limited over such a short period. This is an additional way of reducing the noise affecting the SSB estimation. A similar noise reduction cannot be obtained with collinear differences since the minimum time lag between differenced measurements is one repeat cycle (i.e., 10 days for TOPEX/ POSEIDON).

\subsection{Other Noise Components}

For all altimetric missions before TOPEX/POSEIDON, the radial orbit error $\varepsilon_{H}$ was a major component of the altimetric error budget, ranging typically from several meters to several decimeters RMS. Accordingly, nearly all previous empirical estimates of the SSB were obtained after orbit error reduction. The classic method for removing this error is to fit a model of the orbit error to the data using linear least squares techniques. The model-estimated error is then removed. Orbit error models have been gradually improved, evolving from low-order polynomials fitted to short arcs to Fourier expansions of the theoretical orbit perturbations fitted over very long arcs (for a detailed analysis see Tai and Fu [1986], Engelis [1987], Schrama [1989], or Chelton and Schlax [1993]). Still, data-adjusted orbit errors retain some nonorbital signals, including ocean signals. This affects any subsequent analysis of the data and in particular SSB studies. Zlotnicki et al. [1989] observe significant systematic changes in the estimated sea state bias when changing the length of the arcs on which the orbit error is adjusted. Conversely, Ray and Koblinsly [1991] notice important variations of the computed orbit correction depending on the chosen SSB correction.

Such problems are difficult to avoid when orbit corrections are actually performed. Any data-adjusted orbit error will contain a number of nonorbital signals that can be correlated with SSB. Such correlations will then affect SSB estimations, as observed by Zlotnicki et al. [1989]. However, there is no physical basis for a correlation between SSB and the true orbit error. Accordingly, the use of uncorrected data should yield unbiased SSB estimates, but with larger formal estimation errors. Recently, Gaspar et al. [1992] obtained reasonably accurate estimates of the Geosat SSB without correcting for an orbit error. The GEM-T2 orbit [Haines et $a l ., 1990]$ was used with an estimated radial error close to 40 $\mathrm{cm}$ RMS. The TOPEX/POSEIDON orbit error is an order of magnitude smaller [e.g., Nouel et al., this issue]. It is thus safe, simple, and accurate to avoid orbit correction in this study.

The last noise component in (5) is the altimetric measurement error. The situation is again favorable since every effort was made to minimize the TOPEX/POSEIDON measurement error budget. Errors of the tide model, with an amplitude of about $5 \mathrm{~cm}$ RMS, are presently the largest component in $\varepsilon_{h_{a}^{\prime}}$. This error shall be significantly reduced when new models, calibrated with TOPEX/POSEIDON data, become available. However, little correlation is expected between SSB and tide errors so that these errors should have little impact on the estimated bias.

A bit more worrisome is the inverted barometer (IB) correction which is known to be imperfect and highly correlated with SSB. The correlation between SWH and surface atmospheric pressure is such that empirical estimates of the SSB have the wrong sign (i.e., the estimated bias is toward the wave crests) when the IB correction is not applied to the altimeter data [Gaspar et al., 1992]. Still, a recent analysis of the TOPEX/POSEIDON data by $F u$ and Pihos [this issue] shows that the IB correction is appropriate at latitudes greater than 20 degrees. Significant deviations from a perfect IB response are observed only at lower latitudes. However, the pressure variability is so weak in these latitudes ( 1 to 2 mbar RMS) that errors induced by a possibly inaccurate IB correction remain very small. The IB correction is thus systematically applied in this work. It will be shown in section 4.8 that IB errors in the equatorial ocean have little impact on our global estimates of the SSB.

\subsection{SSB Estimation Using Linear Regression}

Estimation of the SSB from (5) is simple if a linear model of the bias is assumed

$$
\mathrm{SSB}=\sum_{i=1}^{p} a_{i} X_{i}+\varepsilon_{\mathrm{SSB}}
$$

where $\varepsilon_{\mathrm{SSB}}$ is the nonmodeled part of the sea state bias, $a_{i}$ coefficients are $p$ parameters to be estimated and $X_{i}$ variables are SSB-related variables, such as SWH, wind speed $(U)$, backscatter coefficient $\left(\sigma_{0}\right)$, or any combination thereof. Equation (5) then becomes

$$
\Delta \mathrm{SSH}_{m}^{\prime}=\sum_{i=1}^{p} a_{i} \Delta X_{i}+\Delta \varepsilon_{\mathrm{SSB}}+\Delta \eta-\Delta \varepsilon_{H}+\Delta \varepsilon_{h_{i}^{\prime}}
$$

Lumping all errors together as the sum of a zero-mean noise ( $\varepsilon)$ and a bias $\left(a_{0}\right)$, this can be rewritten

$$
\Delta \mathrm{SSH}_{m}^{\prime}=\sum_{i=0}^{p} a_{i} \Delta X_{i}+\varepsilon
$$

where $\Delta X_{0}$ is a dummy variable equal to unity. We thus have a classic multivariate linear regression problem. Given $n$ observations of $\left(\Delta \mathrm{SSH}_{m}^{\prime}, \Delta X_{i}\right)$ and using bold characters to 
denote observations vectors or matrices, the standard linear least squares estimate of the parameter vector $a$ is [e.g., Liebelt, 1967]

$$
\hat{\mathbf{a}}=\left(\Delta \mathbf{X}^{T} \Delta \mathbf{X}\right)^{-1} \Delta \mathbf{X}^{T} \Delta \mathbf{S S H} \mathbf{m}^{\prime}
$$

The estimator is unbiased if $\mathbf{\Delta X}$ and $\varepsilon$ are uncorrelated. It minimizes the sample variance of the residuals $\varepsilon$, or equivalently the variance of $\Delta S_{S H}$, the sea surface height crossover differences corrected for the SSB. Provided the residuals are uncorrelated random variables with common variance $\sigma^{2}$, the covariance matrix of $\mathbf{a}$ is

$$
\mathbf{C}_{\mathbf{a}}=\sigma^{2}\left(\Delta \mathbf{X}^{T} \Delta \mathbf{X}\right)^{-1}
$$

For uncorrelated residuals, an unbiased estimate of $\sigma^{2}$ is obtained by dividing the residual sum of squares by its degrees of freedom, that is, the number of cases minus the number of model parameters. In practice, the residuals in (8) always remain somewhat correlated (less for crossover differences than for collinear differences). The number of degrees of freedom is thus less than theoretically estimated. Accordingly, (10) generally underestimates the true variance of the estimator. Better estimates can be obtained from replication methods or bootstrapping [e.g., Efron, 1979]. The basic idea of these methods is to derive the pertinent statistics concerning the regression coefficients from a population of coefficient estimates obtained from a number of different data subsets. We will use such a technique implemented as follows. Our global data set includes 29 repeat cycles of the TOPEX/POSEIDON mission (see next section). It will be split into subsets containing each the data from one cycle. Regressions will be performed with both the global data set and individual cycles. The coefficient estimates will be obtained from the regression with the global data set. Their uncertainties will be evaluated by computing the standard deviation of the estimates obtained for individual cycles.

\section{Data Sets}

Here use is made of the crossover data sets provided by AVISO. These are based on the TOPEX/POSEIDON merged geophysical data records (GDRs). They are produced operationally, cycle per cycle, and made available on AVISO CD-Roms [AVISO, 1993]. Data from repeat cycle 2 to 30 are employed. They cover the period October 4, 1992 , to July 28, 1993. The Centre National d'Etudes Spatiales (CNES) orbit, based on Doppler orbitography and radiopositioning integrated by satellite (DORIS) measurements, is used for all data. The following standard corrections are applied to the TOPEX (Ku band) and POSEIDON altimeter range measurements: dry tropospheric range delay and inverted barometer effect from European Centre for Medium-Range Weather Forecasts sea level pressure, wet tropospheric range delay from TOPEX microwave radiometer measurements, ionospheric corrections from filtered dualfrequency altimeter measurements for TOPEX and from DORIS measurements for POSEIDON, solid Earth tide, ocean tide from the Cartwright and Ray [1990] model. The wind speed $U$ is deduced from the backscatter coefficient $\left(\sigma_{0}\right)$ using the Modified Chelton-Wentz (MCW) algorithm [Witter and Chelton, 1991a]. The TOPEX backscatter coefficient is corrected for its known bias of $0.7 \mathrm{~dB}$.

Rather stringent editing is applied to eliminate anomalous
Table 1. Number of TOPEX/TOPEX (TT), POSEIDON/ POSEIDON (PP), or TOPEX/POSEIDON (TP) Validated

\begin{tabular}{|c|c|c|c|}
\hline Cycle & $\mathrm{TTT}$ & $\mathrm{PP}$ & $\mathrm{TP}^{\mathrm{P}}$ \\
\hline 2 & 3130 & 0 & 0 \\
\hline 3 & 3229 & 76 & 1020 \\
\hline 4 & 4017 & 139 & 1474 \\
\hline 5 & 4046 & 53 & 909 \\
\hline 6 & 3530 & 220 & 1696 \\
\hline 7 & 5130 & 0 & 0 \\
\hline 8 & 2994 & 95 & 1102 \\
\hline 9 & 3360 & $\cdot 170$ & 1521 \\
\hline 10 & 6403 & 0 & 0 \\
\hline 11 & 5141 & 63 & 1102 \\
\hline 12 & 4214 & 233 & 1891 \\
\hline 13 & 5208 & 115 & 1613 \\
\hline 14 & 4909 & 175 & 1779 \\
\hline 15 & 5567 & 83 & 1407 \\
\hline 16 & 4450 & 84 & 1167 \\
\hline 17 & 7437 & 0 & 0 \\
\hline 18 & 7324 & 0 & 0 \\
\hline 19 & 7166 & 0 & 0 \\
\hline 20 & 0 & 5980 & 0 \\
\hline 21 & 7138 & 0 & 0 \\
\hline 22 & 7050 & 0 & 0 \\
\hline 23 & 6643 & 0 & 0 \\
\hline 24 & 6493 & 0 & 0 \\
\hline 25 & 6374 & 0 & 0 \\
\hline 26 & $62 ! 2$ & 0 & 0 \\
\hline 27 & 5949 & 0 & 0 \\
\hline 28 & 5827 & 0 & 0 \\
\hline 29 & 5594 & 0 & 0 \\
\hline 30 & 5485 & 0 & 0 \\
\hline Total & 150,020 & 7486 & 16,681 \\
\hline
\end{tabular}
Crossover Data for Each Repeat Cycle

data. In particular, all cases with satellite attitude angle larger than $0.3^{\circ}$ are eliminated. Also discarded are measurements with missing or unreliable standard corrections or with $\sigma_{0}<7 \mathrm{~dB}, \sigma_{0}>20 \mathrm{~dB}$, or SWH $>11 \mathrm{~m}$. A complete description of the editing procedure is given by Le Traon et al. [this issue]. After quality control a total of 174,187 crossover differences are obtained, including 150,020 crossover points with TOPEX measuring on both the ascending and the descending arc (these will be referred to as TT crossovers), 7486 crossovers with POSEIDON measuring on both arcs (PP crossovers) and 16,681 crossovers with TOPEX and POSEIDON measuring on one arc each (TP crossovers). The number of TT, PP, or TP crossovers in each cycle is given in Table 1. We use the TT and PP data sets separately to estimate the TOPEX and POSEIDON sea state biases. The results are described in the next two sections.

\section{TOPEX Sea State Bias}

\subsection{Constant Relative Bias}

The adimensional ratio SSB/SWH is often referred to as the relative bias. The simplest, most usual, parameterization of SSB is based on the assumption of a constant relative bias

$$
\mathrm{SSB}_{m}=a_{1} \mathrm{SWH}
$$

This is obviously derived directly from (1) assuming $\lambda_{2}$ to be a constant. The constant $a_{1}$ has been estimated by many authors. For studies based on Geosat measurements the published values typically range from -0.01 to -0.04 , that 
is, -1 to $-4 \%$ of SWH [e.g., Ray and Koblinsky, 1991], in agreement with various airborne and tower measurements [e.g., Walsh et al., 1989]. Values in the same range are found for GEOS 3. Significantly higher values (about $-7 \%$ ) are quoted for the Seasat altimeter. They are considered as atypical and generally attributed to peculiar features of the Seasat altimeter tracker and ground processing [Born et al., 1982; Hayne and Hancock, 1982].

The linear regression on the global (cycle 2 to 30) TT crossover data set yields $\hat{a}_{1}=-0.02$, a very reasonable result close to the typical Geosat estimates. The uncertainty of that estimated coefficient is \pm 0.003 . As explained in section 2.4, this uncertainty is the standard deviation of the different $a_{1}$ estimates obtained by performing regressions on individual cycles. In this case, the computation is based on 28 cycles since our 29-cycle data set includes one subset (cycle 20) without TT crossovers.

Before SSB correction, the variance of the TT crossover differences is $127.71 \mathrm{~cm}^{2}$. After correction the variance is reduced to $120.38 \mathrm{~cm}^{2}$, corresponding to a sea level variability signal of $11 \mathrm{~cm}$ RMS. The variance of the crossover differences explained by this SSB parameterization is thus $7.33 \mathrm{~cm}^{2}$, or $2.7 \mathrm{~cm}$ RMS.

We also analyzed the regression residuals $(\varepsilon)$ as a function of the regressor $\triangle \mathrm{SWH}$. To do so, residuals were sorted into bins $1 \mathrm{~m}$ wide according to $\Delta S W H$. The mean residual within each bin is plotted in Figure 1. The averaged residuals show coherent variations with $\triangle \mathrm{SWH}$. This obviously indicates that the simple SSB parameterization (11) does not account for the whole sea state related variability in the altimeter measurements. In other words, the SSB is not simply linearly dependent on SWH. This is nothing new. Several authors have proposed a variety of more elaborate SSB models to compensate for the shortcomings of (11) [e.g., Ray and Koblinsky, 1991; Melville et al., 1991; Glazman et al., 1994]. We examine such models in the next two subsections. We first analyze the ability of a relatively simple physically based SSB model proposed by $F u$ and Glazman [1991]. We then investigate a whole range of more empirical models, including that selected by NASA to produce the TOPEX GDRs [Callahan, 1992].



Tigure 1. Mean regression residuals for SSB parameterization (11) as a function of $\triangle S W H$. The dots show averages computed on 1-m-wide $\Delta S W H$ bins.



Figure 2. Variance explained by the $F_{u}$ and Glazman [1991] SSB model (12) as a function of the selected value of parameter $d$.

\subsection{Fu and Glazman Model}

The statistical properties of the sea surface, and hence the SSB, depend not only on SWH but also on the degree of wave development [Glaz,man and Pilorz, 1990; Glazman and Srokosz, 1991; Minster et al., 1992]. Glazman and Srokosz [1991] showed that the $\lambda_{2}$ parameter in (1) could be expressed as a function of wave age. Fu and Glazman [1991] (hereinafter referred to as FG) then introduced the concept of pseudo wave age, a proxy for the true wave age which cannot be determined from altimeter data alone. They proposed the following SSB parameterization:

$$
\mathrm{SSB}_{m}=a_{1} \mathrm{SWH}\left(g \mathrm{SWH} / U^{2}\right)^{-d}
$$

where the adimensional ratio $\left(g \mathrm{SWH} / U^{2}\right)$ is the pseudo wave age, with $g$ the acceleration due to gravity; $a_{1}$ and $d$ are empirical parameters to be determined. For Geosat, FG estimated $a_{1}=-0.01$ and $d=0.55$. These estimates were obtained by minimizing collinear differences along selected ground tracks. More recently, Glazman et al. [1994] revised their estimates using a more comprehensive global data set. They found $a_{1}=-0.014$ and $d=0.35$. The FG SSB model is used in the POSEIDON GDRs [Zanife, 1992] and in the AVISO merged GDRs [AVISO, 1993]. The selected parameter estimates are close to those of FG: $a_{1}=-0.01$ and $d=$ 0.5 .

Simultaneous estimation of $a_{1}$ and $d$ is, of course, a nonlinear problem. It is easily solved using iterative minimization methods for which standard computer routines are available. On the other hand, if a value of $d$ is selected, finding the optimal value of $a_{1}$ is a simple linear problem, immediately solved using (9). We actually solved it for a set of regularly sampled values of $d$ in the range $[-0.5,1.5]$. Each experiment gave us the amount of variance explained for one value of $d$. The results are plotted in Figure 2. After this first set of experiments, it was simple to refine our estimate of $d$ in the range of values where the explained variance was the largest. The optimum was found for $\hat{d}=$ 0.17 and $\hat{a}_{1}=-0.016$. Regressions performed on individual cycles, keeping $\hat{d}=0.17$, yield estimates of $a_{1}$ between -0.01 and -0.02 .

For the global TT data set, the variance explained by (12) 
is $7.98 \mathrm{~cm}^{2}$ or $2.8 \mathrm{~cm}$ RMS, a value close to that obtained with a constant relative bias (11). This is not surprising as the optimal value of $d$ is rather close to zero, the value for which (11) and (12) are identical. Accordingly, the regression residuals obtained with the $\mathrm{FG}$ parameterization vary with $\triangle S W H$ in a way that is very similar to that observed in Figure 1. This disappointing result shows that for TOPEX (12) does not extract significantly more SSB-related variability from the crossover differences than (11) does. This is unlike the results previously obtained for Geosat by $\mathrm{FG}$ and Glazman et al. [1994]. Those works yield estimates of $d$ ranging from 0.35 to 0.55 . Figure 2 shows that for TOPEX, exponents in this range yield similar or poorer results than a simple constant relative bias $(d=0)$. This difference between TOPEX and Geosat is as yet unexplained. It might be due to technological differences between the two instruments, but it might also, at least partly, be due to different data processing. In particular, FG and Glazman et al. [1994] use an orbit error correction scheme that might affect the estimated SSB, as previously explained in section 2.3. No orbit adjustment is performed here. There might also be a problem with the wind speed algorithms since Glazman et al. [1994] use the Glazman and Greysukh [1993] wind speed algorithm instead of MCW. To check this we performed new regressions using wind speeds computed with the Glazman and Greysukh [1993] algorithm. The results prove to be only slightly different. The best SSB calibration is obtained for $\hat{d}=0.14$ and $\hat{a}_{1}=-0.017$. The explained variance is 7.87 $\mathrm{cm}^{2}$. It is thus clear the choice of wind speed algorithm cannot be the main cause of the observed differences.

\subsection{Formulation and Selection of Other Empirical Models}

As the physical basis to produce a generally applicable SSB model is lacking, one can, on statistical bases, try to find the relationship that best matches the sea state-related variability of the sea surface height. The theoretical expression (1) shows that SSB is proportional to SWH but the proportionality factor is not exactly known. It is not a constant, as shown in section 2.1, but rather a function of different sea state parameters that remains to be determined. That function can, of course, be approximated with a Taylor series expanded in terms of pertinent sea state related variables. This is the solution adopted to estimate the SSB in the TOPEX GDRs produced by NASA [Callahan, 1992]. In practice, the variables of the Taylor series can only be chosen from sea state related parameters that are measurable by an altimeter: $\mathrm{SWH}, \sigma_{0}, U$, or any combination thereof. In the NASA GDRs use is made of SWH, $U$ and $\rho=\left(r \text { SWH } / U^{2}\right)^{-0.5}$, a wave age related parameter. Simultaneous use of the two highly correlated variables $U$ and $\sigma_{0}$ is purposely avoided. In view of the limited success obtained in the preceding section with the FG parameterization, we will also avoid using $\rho$. We are thus left with two pertinent parameters: $S W H$ and $U$. Like in the NASA GDRs, the series expansion will be limited to the secondorder terms. Adding more terms is unnecessary, as will be shown later. The general SSB formulation to investigate is thus

$\mathrm{SSB}_{m}=\mathrm{SWH}\left[a_{1}+a_{2} \mathrm{SWH}+a_{3} U+a_{4} \mathrm{SWH}^{2}+a_{5} U^{2}\right.$

$$
\left.+a_{6} \text { SWH } U\right]
$$

This relation is obviously linear. All parameters are easily estimated using (9). As usual in regression analysis, we will test models with a progressively increasing number of terms (parameters) in (13). The results obtained with $a_{1}$ as a single parameter were examined in section 2.1. The variance explained is $7.33 \mathrm{~cm}^{2}$. Just to have an idea of the maximum variance one can hope to explain with a model like (13), we performed the full six-parameter regression. The variance accounted for is $10.48 \mathrm{~cm}^{2}$ (3.24 cm RMS). The difference with respect to the simple one-parameter model is thus relatively small. In all following experiments we will keep the zero-order term $a_{\text {, }}$ which turns out to explain a large fraction of the variance. When keeping this term, the number of possible models with $2,3,4,5$, and 6 parameters is 5,10 , 10,5 , and 1 , respectively. This represents a maximum of 31 models to test. To discriminate between all these models we will use three well-defined criteria. For a given number of parameters, the best model is the one (1) which explains the largest amount of variance, (2) whose parameter estimates are most robust (i.e., exhibit the smallest cycle to cycle variability), and (3) whose residuals display the smallest possible coherent variations (i.e., correlations) with the regressors $\Delta S W H$ and/or $\Delta U$. For the ease of notation, each of the selected bias model will be denoted $B M x$, where $x$ is the number of parameters fitted in the model. The constant relative bias model (11) will accordingly be identified as BM1.

\subsection{Two-Parameter Models}

The variance explained by all two-parameter models is shown in Table 2. Among these, two solutions are clearly ahead. These are the models using $\mathrm{SWH}^{2}$ or $\mathrm{SWH}^{3}$ as the second regressor. The explained variance is almost exactly 9 $\mathrm{cm}^{2}$ in both cases. Note that the FG model, also a (nonlinear) two-parameter model, is placed fourth with an explained variance of $7.98 \mathrm{~cm}^{2}$. The Ray and Koblinsky [1991] type of model, with SWH and $U$ SWH as regressors, performs less well. Interestingly, the two best models are functions of SWH only, that is, they do not require wind speed estimation. This can be useful in the early phases of altimeter missions when the $\sigma_{0}$ calibration and wind speed algorithms are not yet ascertained.

It is quite easy to choose between the best two twoparameter models as the solution including $S \mathrm{SH}^{2}$ is clearly less robust. The estimates of $a_{1}$ and $a_{2}$ are strongly anticorrelated and variable. The estimates of $a_{1}$ determined from individual repeat cycles range from -7 to $-3.8 \%$ of SWH. Significantly more stable results are obtained with $\mathrm{SWH}^{3}$ as the second regressor. Our preferred two-parameter model (BM2) thus reads

$$
\mathrm{SSB}_{m}=\mathrm{SWH}\left[a_{1}+a_{4} \mathrm{SWH}^{2}\right]
$$

Figure 3 shows the relative biases corresponding to (14) successively fitted over the 28 individual repeat cycles of our TT crossover data set. The match between all cycles is indeed remarkable. The coefficient estimates are $\hat{a}_{1}=$ $-0.037 \pm 0.004$ and $\hat{a}_{4}=0.00029 \pm 0.00007$. The corresponding relative bias is plotted in Figure 4 .

The basic effect introduced by the $\mathrm{SWH}^{3}$ term in BM2 is a progressive decrease of the magnitude of the relative bias when SWH increases. Interestingly, a similar effect is predicted by the theoretical EM bias model of Barrick and Lipa 
Table 2. Variance of the TT Crossover Differences Explained by Two- and Three-Parameter SSB Models

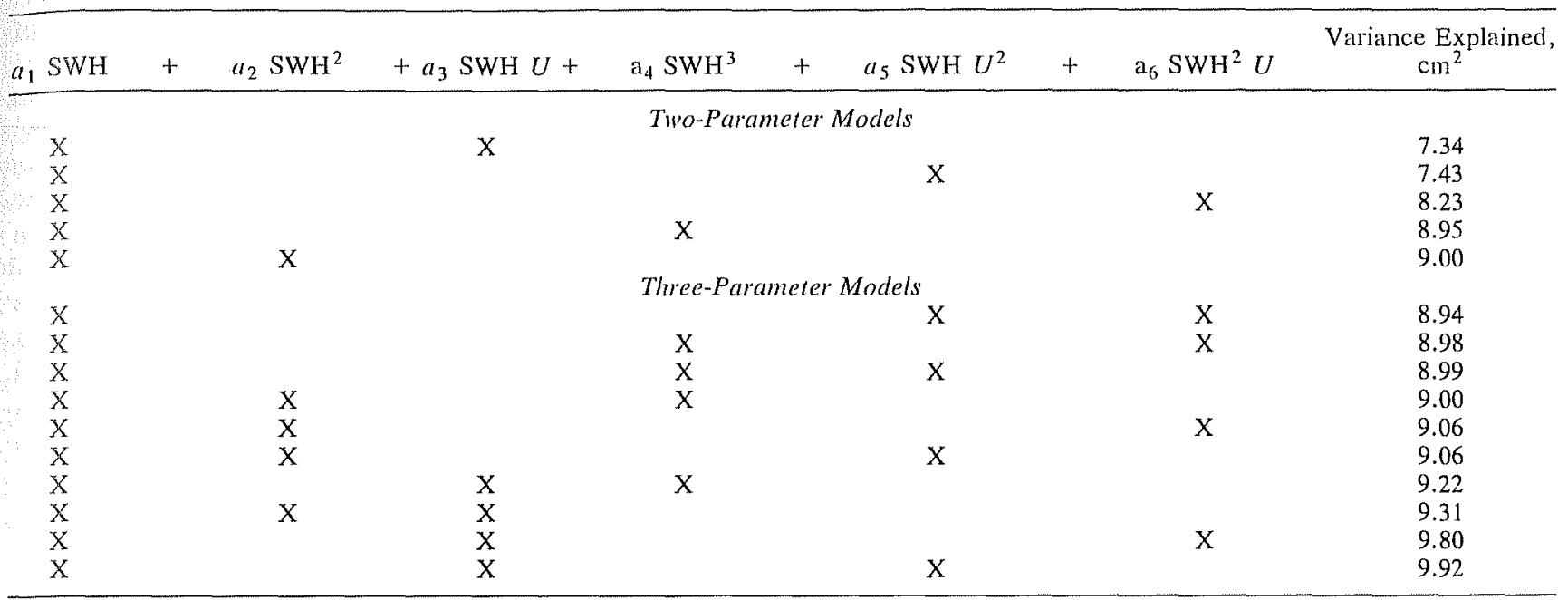

Crosses indicate the terms that are retained in each model. The simple one-parameter model explains $7.32 \mathrm{~cm}^{2}$ and the full six-parameter model explains $10.48 \mathrm{~cm}^{2}$.

[1985]. This model yields a relative bias whose magnitude is proportional to $\mathrm{SWH}^{-0.28}$, clearly a decreasing function of SWH. This result is obtained assuming a Joint North Sea Wave Project directional wave spectrum [Hasselmann et al., 1980]. The simpler theoretical model of Jackson [1979] does not provide conclusive results on that point. This model assumes a one-dimensional Phillips [1977] wave spectrum. Relative bias is shown to be directly proportional to the ocean surface skewness. But no simple correlation is found between skewness and SWH [Rodriguez and Chapman, 1989].

On the other hand, Witter and Chelton [1991b] have also observed a decreasing relative bias with increasing wave heights in the Geosat data. For SWH values below $3 \mathrm{~m}$ they find nearly constant relative biases between -3 and $-4 \%$ of SWH, as in BM2 (see Figure 4). The decrease in the magnitude of the relative bias becomes well marked for SWH $>3 \mathrm{~m}$. Witter and Chelton [1991b] attribute this effect to attitude and sea state errors in the Geosat altimeter

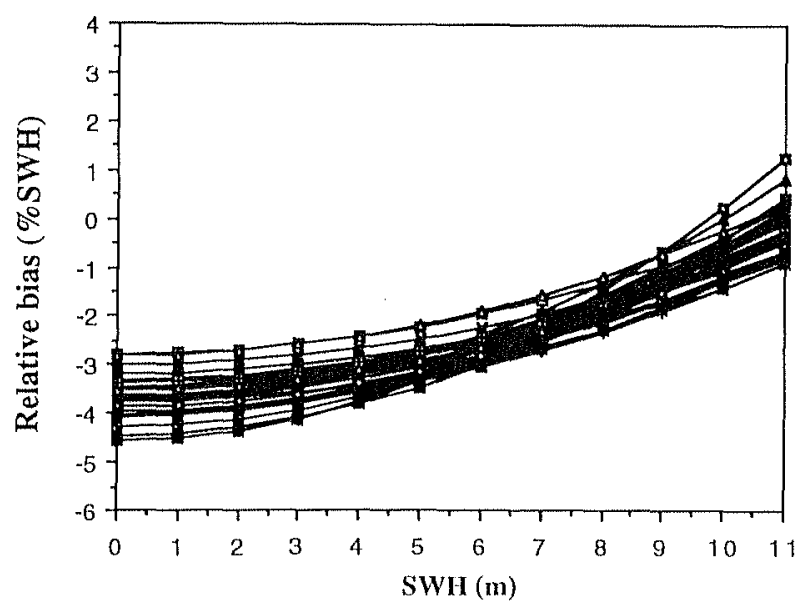

Figure 3. Relative bias estimates as a function of SWH. These estimates are obtained by fitting BM2 (14) over 28 different TT crossover data subsets, each corresponding to one repeat cycle (cycles 2 to 19 and 21 to 30 ). on-board tracking algorithm. Here we think it is more likely to be a true sea state bias effect. Indeed, TOPEX/ POSEIDON has never had Geosat's severe attitude problems. Small pointing errors (generally below $0.4^{\circ}$ ) were present until the end of cycle 8 . After that, pointing is almost perfect (error below $0.1^{\circ}$ ). Attitude errors are thus unlikely to be present in the estimated sea state biases of cycle 9 to 30. Since our bias estimates before and after cycle 8 are very comparable, there is probably no attitude effect at all in our results.

\subsection{Three-Parameter Models}

Turning now to three-parameter models, it appears that most of the 10 tested models do not explain significantly more variance than BM2. Interestingly, the four best models all include the SWH $U$ term. The best one (BM3) reads

$$
\mathrm{SSB}_{m}=\mathrm{SWH}\left[a_{1}+a_{3} U+a_{5} U^{2}\right]
$$

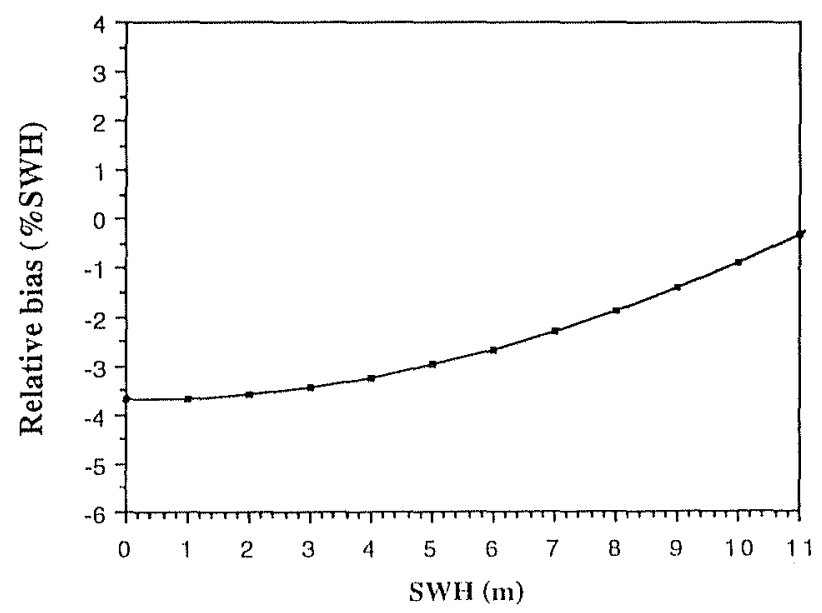

Figure 4. Relative bias estimate as a function of SWH. This estimate is obtained by fitting BM2 (14) to our global TT crossover data set. 


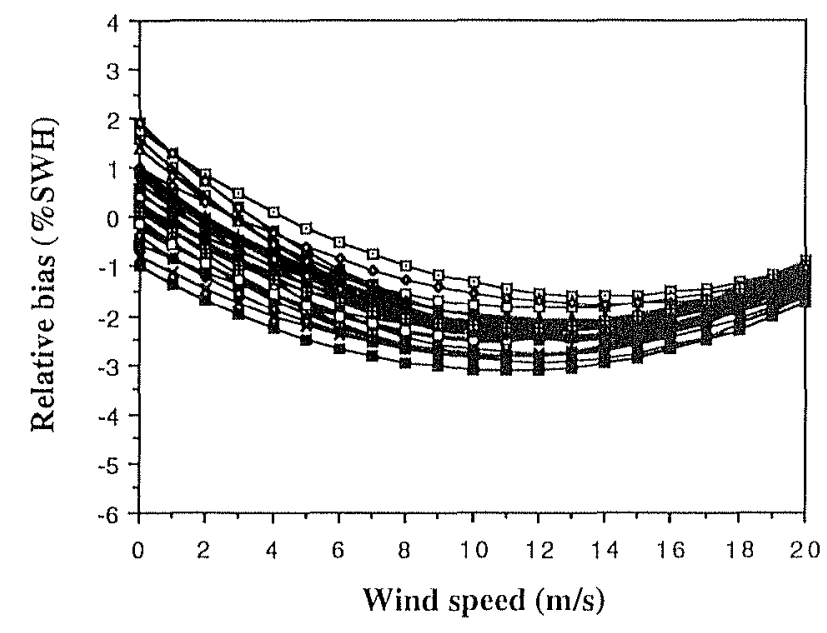

Figure 5. Relative bias estimates as a function of $U$. These estimates are obtained by fitting BM3 (15) over 28 different TT crossover data subsets, each corresponding to one repeat cycle (cycles 2 to 19 and 21 to 30 ).

The relative bias is, noticeably, a function of wind speed only. This solution explains a variance of $9.92 \mathrm{~cm}^{2}$. The coefficient estimates are $\hat{a}_{1}=0.0036 \pm 0.007, \hat{a}_{3}=$ $-0.0045 \pm 0.0008$, and $\hat{a}_{5}=0.00019 \pm 0.00003$. Regressions performed on individual cycles indicate that this solution is quite robust (Figure 5). Still, the relative bias estimates are slightly more variable than those obtained with BM2, specially at low wind speeds. This was to be expected since increasing the number of model parameters generally increases the variance of the parameter estimates. BM3 was actually implemented by NASA to produce the TOPEX GDRs. The estimated parameter values are [Callahan, 1992] $\hat{a}_{1}=-0.0029, \hat{a}_{3}=-0.0038$, and $\hat{a}_{5}=0.000155$. These parameter estimates are remarkably close to ours. The corresponding explained variance is $9.3 \mathrm{~cm}^{2}$. Figure 6 shows that the NASA parameterization yields relative biases that are slightly below those obtained here but the difference always remains smaller than $0.7 \%$ for wind speeds up to 20 $\mathrm{m} / \mathrm{s}$.

The NASA algorithm selection was based on Hevizi et al.'s [1993] extensive analysis of recent tower and airborne measurements. Interestingly, their data set included new observations made at high wind speeds, up to $20 \mathrm{~m} / \mathrm{s}$. Such measurements were not previously available. As indicated by the authors, these new measurements give confidence that the wind speed dependence of the relative bias is quadratic, not linear as previously considered [e.g., Melville et al., 1991; Ray and Koblinsky, 1991]. This result is clearly confirmed here. The numerical scattering experiments of Rodriguez et al. [1992] also provide theoretical support to that experimental result. Rodriguez et al. [1992] simulations indeed indicate that the magnitude of the relative bias increases with wind speed for wind speeds up to about 10 $\mathrm{m} / \mathrm{s}$, and slightly decreases at higher wind speeds. This is again remarkably consistent with the behavior simulated by BM3.

Now the question arises whether we should prefer a two-parameter model with an explained variance of $9 \mathrm{~cm}^{2}$ or a three-parameter model explaining $9.9 \mathrm{~cm}^{2}$. This is where it is useful to analyze the regression's residuals. As previously done for the regression with BMI, we sorted the residuals for the two- and three-parameter models into $\triangle \mathrm{SWH}$ bins of width $1 \mathrm{~m}$ but also into $\Delta U$ bins of width $1 \mathrm{~m} / \mathrm{s}$. The results are shown in Figures $7 \mathrm{a}, 7 \mathrm{~b}, 8 \mathrm{a}$ and $8 \mathrm{~b}$. The comparison between Figure I and Figure 7a clearly shows that the $\mathrm{SWH}^{3}$ term added to (11) significantly reduces the variations in the residuals as a function of $\triangle \mathrm{SWH}$. All binned residuals now have means below $2 \mathrm{~cm}$. Most are smaller than $1 \mathrm{~cm}$, an excellent result. The mean residuals sorted according to $\Delta U$ are also small but show a very clear trend with $\Delta U$. This is not surprising as (14) does not include any wind-dependent terms while wind-induced variations of the SSB are reported in numerous experiments [e.g., Melville et al., 1991; Walsh et al., 1991; Ray and Koblinsky, 1991; Branger et al., 1993]. This trend is probably not easily explained by one simple wind-dependent term. Indeed, of all the three-parameter models we tested, none of the solutions including SWH and $\mathrm{SWH}^{3}$ plus a wind-dependent term performs significantly better than BM2. The residuals from BM3 behave quite differently: the mean residuals binned according to $\Delta U$ are very small, below $0.5 \mathrm{~cm}$, while the same residuals binned according to $\triangle \mathrm{SWH}$ have significantly larger means, up to $2.5 \mathrm{~cm}$, and show marked SWH-related variations.

To summarize, BM2 and BM3 are two models representing fairly well either the wave-induced SSB variability (BM2) or the wind-induced variability (BM3). As previously mentioned, there is no hope of finding a solution by simply adding one of the wind-dependent terms to (14). This brings us to consider a four-parameter model. Given the very small amount of explained variance one can still gain, the need for such a model can be questioned. However, one must be aware that our data set is dominated by crossover differences associated with wave heights and wind speeds close to the means ( $3 \mathrm{~m}$ and $7.5 \mathrm{~m} / \mathrm{s}$ for $\mathrm{SWH}$ and $U$, respectively). Most of the variance we are trying to explain thus corresponds to "normal" wind and wave conditions. That part of the variance is already well explained by BM1 and, even better, by BM2 and BM3. Figures 1, 7, and 8 indeed show that large residuals are found mostly at high values of $\Delta \mathrm{SWH}$ and $\Delta U$, that is, for measurements including wind speeds or wave heights that deviate significantly from the mean. There

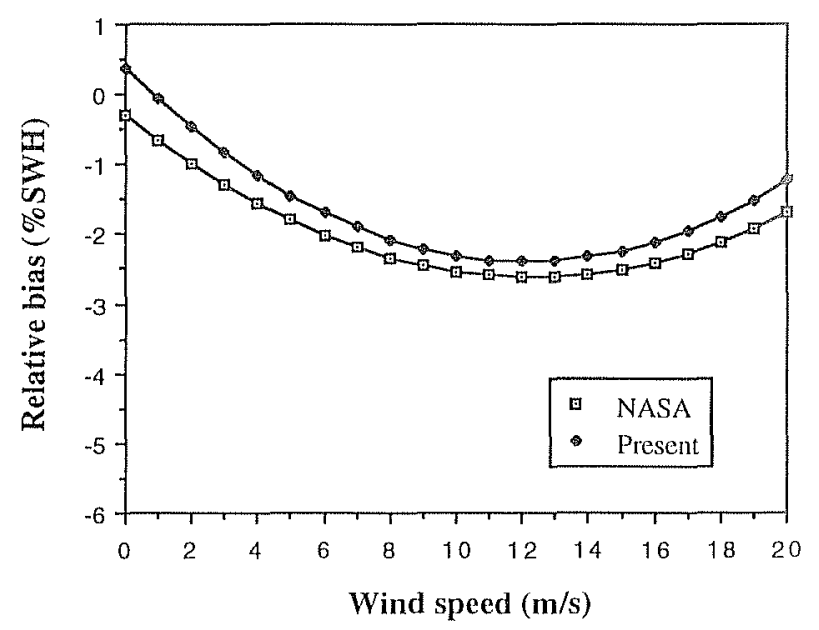

Figure 6. Comparison between the relative bias used in the NASA TOPEX GDRs and the bias obtained by fitting BM3 (15) to our global TT crossover data set. 
are few such measurements, and they therefore make only a small contribution to the total variance. Still, they are most interesting cases from which one can learn about the SSB variability. If a four-parameter model can reduce the residuals in those extreme cases without degrading the solution for normal situations, then we believe it is useful, even if the additional variance explained is very small.

\subsection{Four-Parameter Models}

As previously mentioned, (13) provides 10 possible combinations of four-parameter models. We performed the 10 regressions and found only three models explaining more variance than BM3. They all contain the three basic terms of $\mathrm{BM} 3$ plus one of the other three possible terms: $\mathrm{SWH}^{2}, U$ $\mathrm{SWH}^{2}$, or $\mathrm{SWH}^{3}$. The explained variance is, in the same order: $10.46,10.42$, and $10.41 \mathrm{~cm}^{2}$. The largest explained variance is now extremely close to that obtained with the full six-parameter model $\left(10.48 \mathrm{~cm}^{2}\right)$. The differences are small between the best three models, but the largest explained variance is obtained when including $\mathrm{SWH}^{2}$ as the fourth regressor. This term already provided the best two-
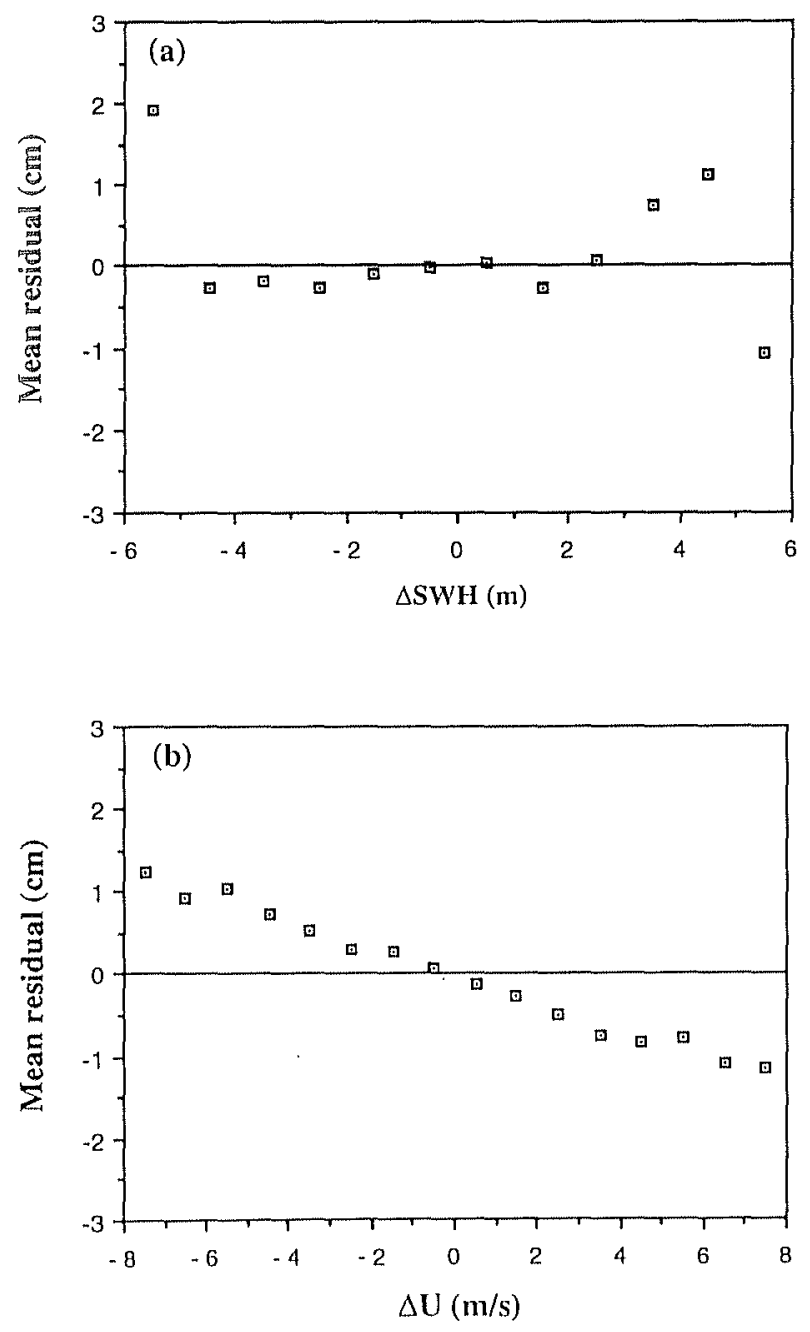

Figure 7. Mean regression residuals for $\mathrm{BM} 2$ (14) as a function of (a) $\triangle \mathrm{SWH}$ and (b) $\Delta U$. The squares show averages computed on $\Delta S W H$ bins of width $1 \mathrm{~m}$ in Figure $7 \mathrm{a}$ and on $\Delta U$ bins of width $1 \mathrm{~m} / \mathrm{s}$ in Figure $7 \mathrm{~b}$.


Figure 8. As in Figure 7, but for BM3 (equation (15)).

parameter model, in terms of explained variance. The best four-parameter model (BM4) thus reads

$$
\mathrm{SSB}_{m}=\mathrm{SWH}\left[a_{1}+a_{2} \mathrm{SWH}+a_{3} U+a_{5} U^{2}\right]
$$

with $\hat{a}_{1}=-0.019 \pm 0.009, \hat{a}_{2}=0.0027 \pm 0.0011, \hat{a}_{3}=$ $-0.0037 \pm 0.0008$, and $\hat{a}_{5}=0.00014 \pm 0.00003$. The relative bias is plotted in Figure 9. The new term actually controls the SWH-related variations of the relative bias whose magnitude now decreases with SWH. This type of effect was already parameterized by BM2 using a term proportional to $\mathrm{SWH}^{3}$. Interestingly, the addition of a $\mathrm{SWH}^{2}$ term to BM3 only has a limited impact on the estimated $a_{3}$ and $a_{5}$ parameters which govern the winddependent response of the SSB. On the contrary, the addition of the new wave-dependent term has a significant impact on $a_{1}$. The estimate of $a_{1}$ in BM3 was a small, but unexpectedly positive, $0.36 \%$ of SWH. The new value obtained with BM4 is $-1.9 \%$ of SWH, an apparently more reasonable SSB estimate for low wind speeds and wave heights.

Analysis of the regression residuals confirms that BM4 performs quite well in a wide range of conditions. The residuals binned according to $\triangle S W H$ generally have means well below $1 \mathrm{~cm}$ (Figure 10a). The means are even smaller when the residuals are sorted according to $\Delta U$ (Figure 10b). 


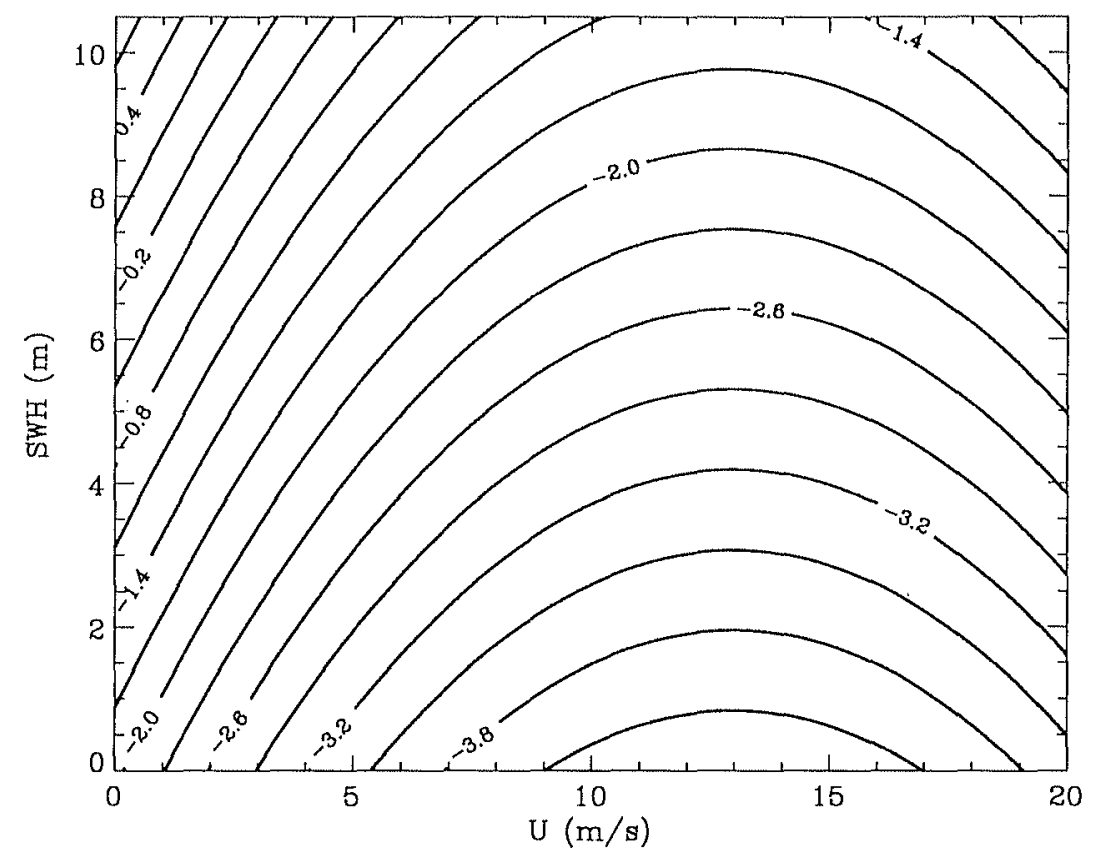

Figure 9. Relative bias estimate $(\% \mathrm{SWH})$ as a function of SWH and $U$. This estimate is obtained by fitting BM4 (16) to our global TT crossover data set.

BM4 thus models the SSB by simply combining the basic wave- and wind-related variations that were separately parameterized in BM2 and BM3. As shown earlier, these basic functional dependences are supported by theoretical developments and experimental results. A linear combination of the two effects is thus physically appealing, but currently there are no theoretical results to justify it. Numerical simulations of the EM bias, such as those performed by Rodriguez et al. [1992], could provide interesting results in that regard. Unfortunately, the simulations presented so far are limited to significant wave heights between 0 and $3 \mathrm{~m}$, a range too narrow to detect any significant SWH-related trend. It is hoped that the empirical results presented here will provide impetus for new studies into the physics of the combined wind and wave effects on sea state bias.

\subsection{Recapitulation}

Given our simple empirical approach, we can hardly expect improvements beyond the four-parameter model. Three generic well-behaved SSB models (BM2, 3, and 4) have been selected for TOPEX data. Their exact expressions read

$\mathrm{BM} 2(\mathrm{~T}): \quad \mathrm{SSB}_{m}=\mathrm{SWH}\left[-0.037+0.00029 \mathrm{SWH}^{2}\right]$

$\mathrm{BM} 3(\mathrm{~T}): \quad \mathrm{SSB}_{m}=\mathrm{SWH}[0.0036-0.0045 U$

$$
\left.+0.00019 U^{2}\right]
$$

$\mathrm{BM} 4(\mathrm{~T}): \quad \mathrm{SSB}_{m}=\mathrm{SWH}[-0.019-0.0037 U$

$$
+0.00014 U^{2}+0.0027 \text { SWH] }
$$

where (T) has been added to the name of each model to identify expressions that have been specifically fitted to TOPEX data. This is to avoid later confusion with similar
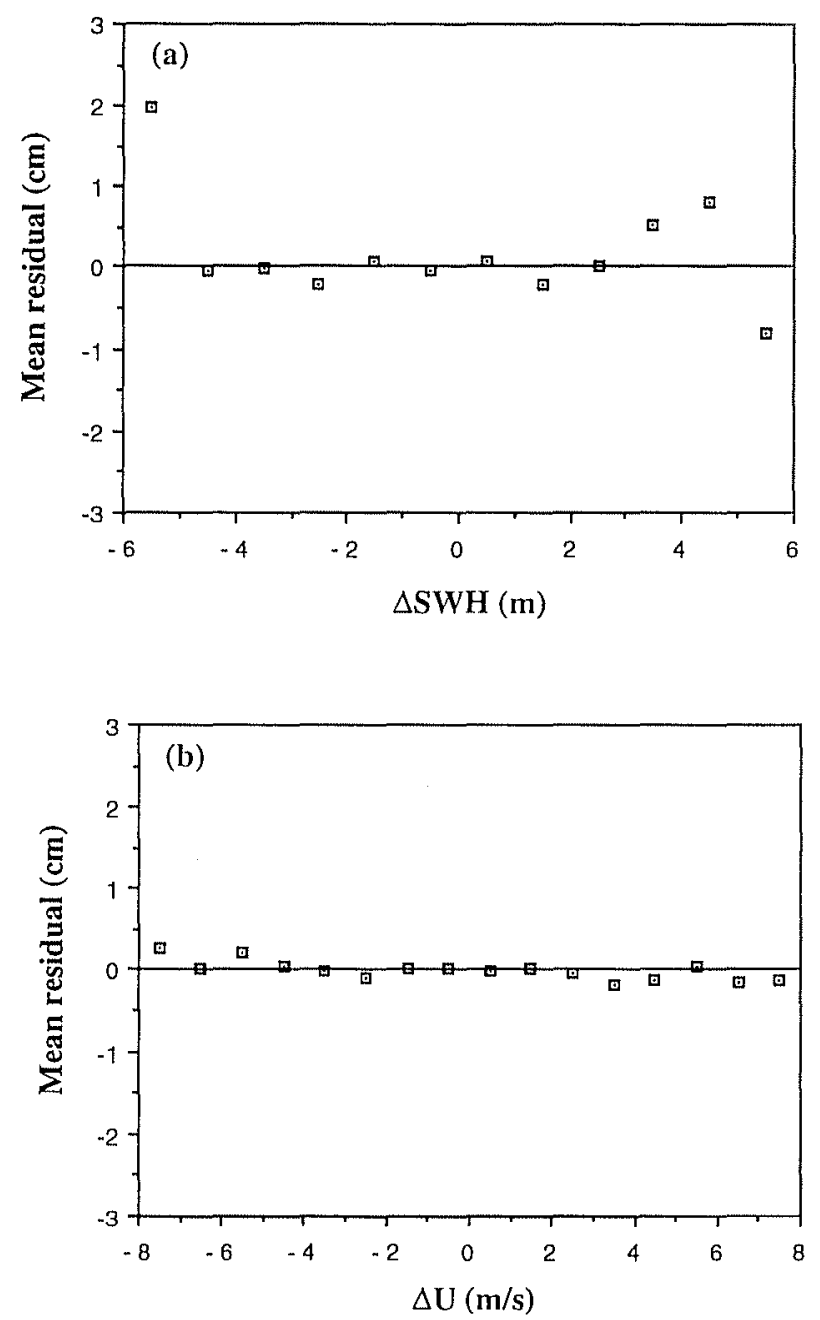

Figure 10. As in Figure 7, but for BM4 (equation(16)). 
results obtained for POSEIDON. Estimated uncertainties for all model coefficients can be found in the text.

\subsection{Regional Performances of the Selected Models}

The selected models have been fitted over global data sets and, accordingly, the models have been classified as a function of the explained variance computed over the globe. A finer examination of the results has been performed through the analysis of regression residuals. These have been computed for different classes of values of $\Delta S W H$ and $\Delta U$. It has been shown that the models with relatively few parameters (BM2, BM3) perform quite well for small values of $\triangle S W H$ and $\Delta U$, generally corresponding to crossover data with near-average wind and wave conditions. BM4 proves to be superior (i.e., has significantly smaller regression residuals) only at large values of $\Delta S W H$ and $\Delta U$, that is, mostly for measurements including wind speeds or wave heights that largely deviate from the global mean. This suggests that BM2 or BM3 could perform nearly as well as BM4 in regions where extreme conditions are rare. More generally one would like to know if the ranking of our globally fitted models still holds at the regional level since the regional wind and wave statistics are largely variable, as shown, for example, in Figures 11a and 11b.

To investigate that, all TT crossover data between $65^{\circ} \mathrm{S}$ to $65^{\circ} \mathrm{N}$ were binned into $10^{\circ}$ latitude bands. The crossover differences and their variances were first computed in each bin without correcting for the SSB. They were then computed using the SSB corrections deduced from BM2(T), $B M 3(T)$, and BM4(T), successively. The crossover differences were also computed assuming a SSB equal to $-2 \%$ of SWH, that is, using the BM1(T) model. Since the variance of both SWH and $U$ increases polewards (see Figures 11a and $11 \mathrm{~b}$ ), the variance explained by all SSB models (BM1 to BM4) also increases toward the poles. The explained variances range from nearly zero at the equator up to 15 or 16 $\mathrm{cm}^{2}$ in midlatitude and high latitudes. The very small explained variance in the equatorial ocean is not surprising. Indeed, the variance of SWH between $5^{\circ} \mathrm{S}$ and $5^{\circ} \mathrm{N}$ is about $0.2 \mathrm{~m}^{2}$. A typical SSB of $-2 \%$ of SWH can thus explain a maximum variance of $0.8 \mathrm{~cm}^{2}$. However, the variance actually explained by BM4 is only $0.2 \mathrm{~cm}^{2}$. The variance explained by $\mathrm{BM} 2$ is even negative. This is probably due to inaccuracies in the IB correction. Indeed, the IB correction is strongly correlated with the SSB [Gaspar et al., 1992] and has significant errors in the equatorial band $[F u$ and Pihos, this issue]. In this region its variance (typically between 1 and $4 \mathrm{~cm}^{2}$ ) is larger than that of the SSB. Errors in the IB correction can thus easily corrupt the weak SSB signal. As a consequence, models that effectively correct for the true SSB can appear to have a negligible or even a negative impact on the explained variance in the equatorial ocean.

The above mentioned meridional variations of the explained variance are common to all models and much larges than the differences between the models. To facilitate the comparison between these models, we computed for each latitude band the differences between the variance explained by BM1 and that explained by the other models. These differences are plotted in Figure 12. Only minor differences (both positive and negative) are observed between BM2 and BM1 in the $\left(20^{\circ} \mathrm{S}, 20^{\circ} \mathrm{N}\right)$ latitude band. This is easily understood as the difference between the two models is a term proportional to $\mathrm{SWH}^{3}$ which becomes significant only for

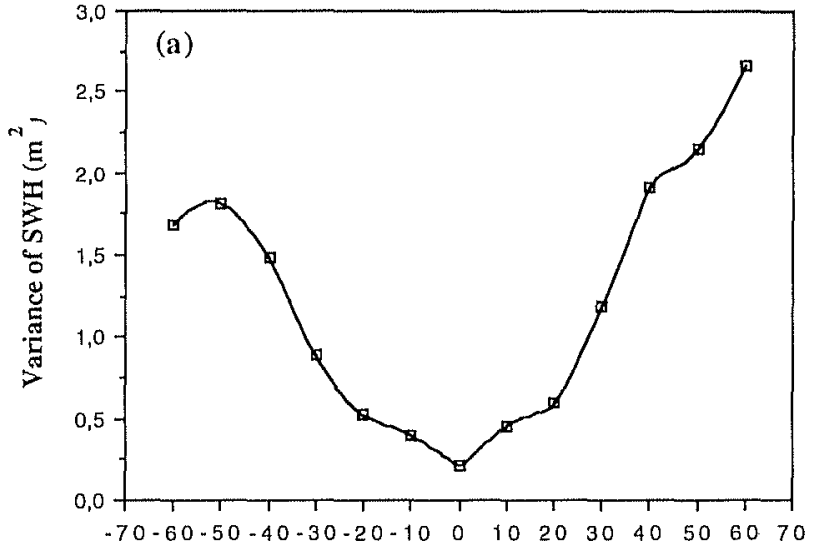

Latitude (degrees)



Latitude (degrees)

Figure 11. Meridional distribution of the variance of (a) the significant wave height and (b) the wind speed, as observed by TOPEX.

large values of the SWH (see Figure 4). Large waves are rare in the tropics: the mean SWH is $1.8 \mathrm{~m}$ with a standard deviation of $0.7 \mathrm{~m}$. The performance of BM2 is significantly better in midlatitudes and high latitudes where larger and highly variable wave heights are observed. BM3 is better than BM2 at all latitudes. The wind speed dependence of the relative bias, as modeled by $\mathrm{BM} 3$, thus clearly explains more variance than the wave height dependence modeled by BM2. However, the combination of the two effects, as modeled in $\mathrm{BM} 4$, proves to be superior to $\mathrm{BM} 3$ at latitudes greater than 30 degrees, in regions of usually higher sea states. The difference between $B M 4$ and $B M 3$ very much resembles that between BM2 and BM1: the effect of the additional SWHdependent term is apparent only in regions where large waves are commonly observed.

The discussion about the results obtained near the equator indicate that calibrating SSB models with data from the equatorial ocean can be misleading. Fortunately, the use of such data proves to be of minor importance in this work. Indeed, the data between $20^{\circ} \mathrm{S}$ and $20^{\circ} \mathrm{N}$ only represent $11 \%$ of our global data set. In addition, the SSB variance in that latitude band is typically 1 order of magnitude smaller than 




Figure 12. Meridional distribution of the variance explained by the BM2, BM3, and BM4 models minus the variance explained by BM1.

that observed at higher latitudes. The impact of the lowlatitude data on our regression results is thus likely to be negligibly small. This was verified by performing new regressions with the BM1 to BM4 models excluding all data within 20 degrees from the equator. Most model coefficients remain virtually unchanged. The largest variations are observed with BM4. The new regression yield is

$\mathrm{SSB}_{m}=\mathrm{SWH}\left[-0.021-0.0037 U+0.00014 U^{2}\right.$

$+0.0028 \mathrm{SWH}]$

Comparison with $(17 \mathrm{c})$ immediately shows that the changes are indeed minor, well within the error bars of the coefficient estimates.

\section{POSEIDON Sea State Bias}

Due to the relatively short period of time during which POSEIDON has been operating from cycle 2 to 30 , our data set only contains 7486 PP crossovers. Estimates of the POSEIDON SSB are thus bound to be more uncertain than for TOPEX. Also, the robustness of the different SSB models can hardly be tested by repeating regressions over different data subsets: our global PP data set is about the size of one TT subset (data from a single repeat cycle)! Still, the general approach used for the TOPEX SSB analysis remains valid and will be repeated for POSEIDON.

\subsection{Constant Relative Bias and FG Model}

The regression based on the simple constant relative bias parameterization (BM1) yields a surprising result: the estimated bias is $-5 \%$ of SWH and the variance explained is $34.55 \mathrm{~cm}^{2}$ (5.9 cm RMS). The variance of the PP crossover differences, before SSB correction, is $160.94 \mathrm{~cm}^{2}(12.7 \mathrm{~cm}$ RMS), significantly larger than for TOPEX $\left(127.71 \mathrm{~cm}^{2}\right.$ or $11.3 \mathrm{~cm}$ RMS). After correction, the variance is reduced to $126.39 \mathrm{~cm}^{2}$ (11.2 cm RMS). This compares better with the TOPEX figure $\left(120.38 \mathrm{~cm}^{2}\right.$ or $11 \mathrm{~cm}$ RMS $)$, obtained after a correction of $-2 \%$ of SWH. There is thus a clear difference of about $3 \%$ of SWH in the sea state-related signal contained in the TOPEX and POSEIDON data. The TOPEX (Ku band) and POSEIDON operating frequencies being nearly identical, no difference is expected between their actual EM biases. The observed difference is thus probably due to differences in the skewness and/or tracker biases of the two altimeters.

The skewness bias can be approximated by $(-\lambda / 24) \mathrm{SWH}$, where $\lambda$ is the skewness of the sea surface elevation [Srokosz, 1986; Rodriguez, 1988]. According to Rodriguez. and Chapman [1989], the average value of $\lambda$ is between 0.2 and 0.3 , implying a skewness bias between -0.8 and $-1.2 \%$ of SWH. The POSEIDON data are not corrected for this bias [Zanife, 1992]. However, even if the TOPEX data were perfectly corrected for it, this bias could only explain SSB differences of about $1 \%$ of SWH between TOPEX and POSEIDON. We are thus left with a difference of, at least, $2 \%$ of SWH which can only be explained by tracker biases. Since the observed TOPEX SSB is too small ( $-2 \%$ of SWH) to include a significant tracker bias, we are led to assume that a tracker bias of $-2 \%$ to $-3 \%$ of SWH is present in the POSEIDON data.

The FG model was also tested using PP data. As for TOPEX, regression series were performed using different values for exponent $d$ in (12). The largest amount of variance $\left(34.72 \mathrm{~cm}^{2}\right)$ is explained for $\hat{d}=0.03$ and $\hat{a}_{1}=-0.049$. With such a small $\hat{d}$ this solution is nearly identical to the simple constant relative bias model. This might indicate that an unusually large fraction of the SSB is modeled well by a constant relative bias. This would be the case if the observed skewness and tracker biases were properly modeled by a constant fraction of SWH.

\subsection{Other Empirical Models}

As for TOPEX, we tested the generic model (13) with gradually more parameters. A total of 25 regressions were performed with all possible two-, three-, and four-parameter models. In these three categories the models that explain the largest amounts of signal variance prove to be exactly the same as for TOPEX, that is, BM2, 3, and 4. Among the two-parameter models, BM2 ranks first, closely followed by the solution including the $\mathrm{SWH}^{2}$ term instead of $\mathrm{SWH}^{3}$. For the three-parameter models, BM3 is once again clearly ahead. In the four-parameter category, BM4 explains the largest amount of variance $\left(35.337 \mathrm{~cm}^{2}\right)$, nearly as much as the full six-parameter model $\left(35.347 \mathrm{~cm}^{2}\right)$. The second and third ranking models are the same as for TOPEX. The BM2, 3 , and 4 models fitted to POSEIDON data read

$\mathrm{BM} 2(\mathrm{P}): \quad \mathrm{SSB}_{m}=\mathrm{SWH}\left[-0.059+0.00016 \mathrm{SWH}^{2}\right]$

$\mathrm{BM}_{3}(\mathrm{P}): \mathrm{SSB}_{m}=\mathrm{SWH}[-0.040-0.0025 U$

$$
\left.+0.00012 U^{2}\right]
$$

$\mathrm{BM} 4(\mathrm{P}): \quad \mathrm{SSB}_{m}=\mathrm{SWH}[-0.047-0.0023 U$

$$
\left.+0.00011 U^{2}+0.0010 \mathrm{SWH}\right]
$$

Due to the relatively small number of available data, the binned residuals for each of these models are considerably noisier than for TOPEX. Still, the mean residuals obtained with the four-parameter model are mostly below $1 \mathrm{~cm}$ (for the same bins as used for TOPEX) and exhibit no $\triangle \mathrm{SWH}$-or $\Delta U$-dependent trends. 


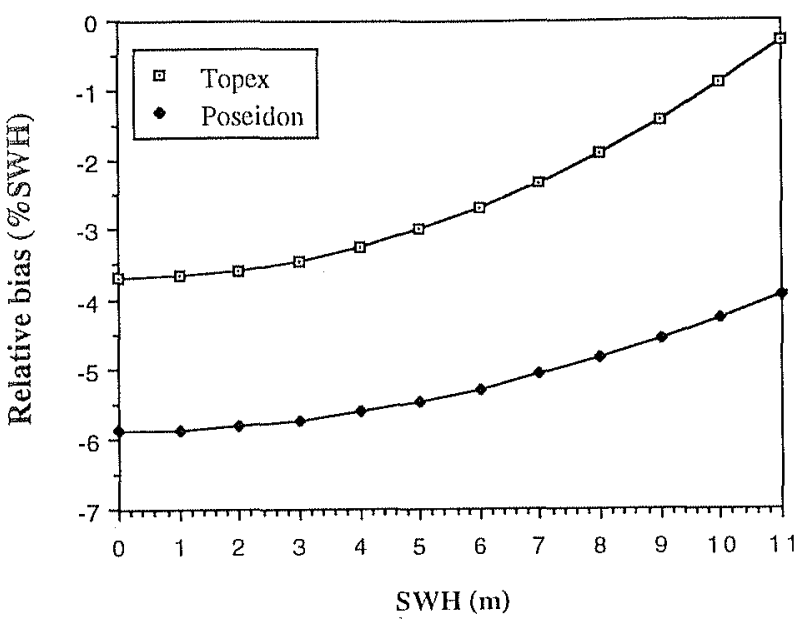

Figure 13. $\mathrm{BM} 2(\mathrm{P})$ and $\mathrm{BM} 2(\mathrm{~T})$ relative biases as a function of SWH.

The relative biases corresponding to $\mathrm{BM} 2(\mathrm{P})$ and $\mathrm{BM} 3(\mathrm{P})$ are plotted in Figures 13 and 14, respectively. They are compared with the corresponding TOPEX models. The difference between the TOPEX and POSEIDON relative biases appears to be nearly constant. The same holds true with the four-parameter models. For a mean SWH of $3 \mathrm{~m}$ and a mean wind speed of $7.5 \mathrm{~m} / \mathrm{s}$, the differences between the TOPEX and POSEIDON BM2, BM3, and BM4 models are $-2.3,-3.2$ and $-2.4 \%$ of $\mathrm{SWH}$, respectively. Though somewhat scattered, these values are consistent with the $-3 \%$ estimate obtained with BM1. Such differences remain too large to be explained by a skewness bias only.

To summarize, the POSEIDON SSB exhibits wind- and wave-related variations that are very close to those observed with the TOPEX altimeter. This variability is probably the natural sea state-related variability of the EM bias. Superimposed on it, a nearly constant relative bias is apparent in the POSEIDON data. It is probably the sum of an uncorrected skewness bias and a tracker bias.

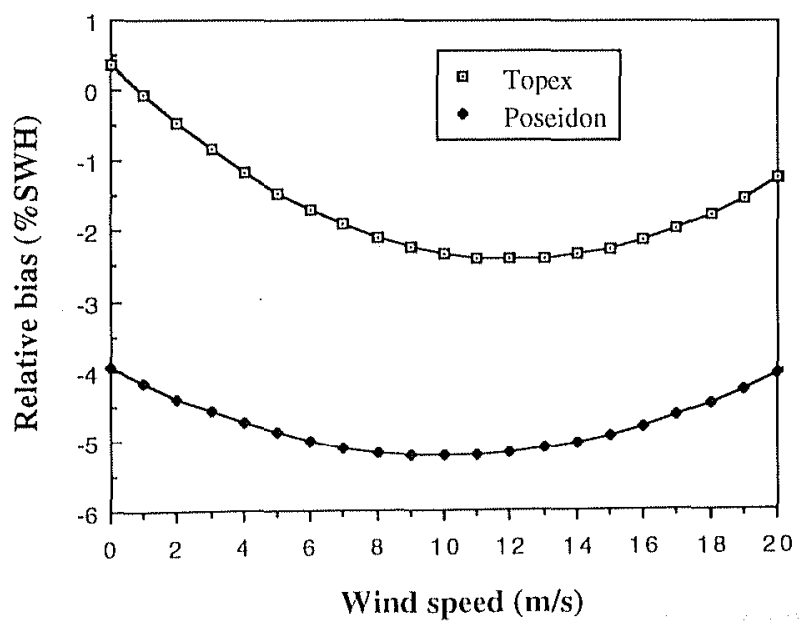

Figure 14. $B M 3(P)$ and $B M 3(T)$ relative biases as a function of $U$.

\section{Conclusion}

A large global data set of TOPEX and POSEIDON crossover differences was used to estimate the SSB of the two altimeters. Data from repeat cycles 2 to 30 are employed. They include over 150,000 TT and 7000 PP crossovers. A simple linear estimation technique is used to extract a modeled SSB signal from the crossover differences. Different SSB models were tested. The experiments performed with TOPEX data indicate the following conclusions:

1. The mean TOPEX sea state bias averaged over all wind and wave conditions is close to $-2 \%$ of SWH.

2. The magnitude of the bias tends to decrease with SWH. This tendency is well reproduced using a two-parameter model (BM2) in which the relative bias (SSB/SWH) is represented by a constant plus a quadratic term in SWH. Such a parameterization outperforms the nonlinear twoparameter model of Fu and Glazman [1991].

3. The wind-induced variations in SSB are modeled well using the three-parameter formula (BM3) presently used in the NASA GDRs. The associated relative bias is a quadratic function of wind speed.

4. To account for both the wind- and wave-related variability of the SSB, a four-parameter model is needed. We found that the most appropriate four-parameter model (BM4) includes the NASA three-parameter model plus a term proportional to $\mathrm{SWH}^{2}$.

5. BM2 proves to be significantly better than a constant relative bias only in midlatitudes and high latitudes where the SWH-induced variability of the relative bias becomes important. BM3 does better than BM2 everywhere. BM4 outperforms BM3 only at latitudes greater than 30 degrees, once again in regions where the SWH-induced variability of the relative bias is significant.

The POSEIDON data set is considerably smaller than the TOPEX data set. All POSEIDON SSB estimates thus have relatively larger uncertainties. However, the data show beyond doubt that the POSEIDON SSB is significantly larger than that of TOPEX. This bias appears to consist of an uncorrected skewness bias plus a tracker bias superimposed on a natural EM bias. Different model fits yield estimates of the skewness + tracker bias ranging from -2.3 to $-3.2 \%$ of SWH for average wind speed and wave height values ( $U=$ $7.5 \mathrm{~m} / \mathrm{s}$, SWH $=3 \mathrm{~m}$ ). The EM bias exhibits wind- and SWH-related variations similar to those observed for TOPEX. The same two-, three-, and four-parameter empirical SSB models explain the largest amount of variance in both the TOPEX and POSEIDON data.

This study has thus produced, for both TOPEX and POSEIDON, a set of empirical SSB models including up to four adjustable parameters. The four-parameter model appears to be robust and to provide good results in a wide range of wind and wave conditions. But this model is empirical. It may include some signal that is only empirically correlated with the SSB. The search for an efficient, physically based, SSB model is thus encouraged. It should take into account both the wind-and the wave-induced variability of the relative bias.

Nearly 16,700 TP crossover data remain unused. They obviously contain information about the TOPEX and, more importantly, POSEIDON SSB. A slight generalization of regression (8) is needed to extract the information. This will be the subject of further work. 
Acknowledgments. Most of the software used in this study was originally developed by El Hassane Makhmara. The authors thank Philippe Escudier, Roman Glazman, Yves Ménard, Jean-François Minster, Emesto Rodriguez, Jacques Stum, and Ed Walsh for helpful discussions and/or comments on this work. Jim Richman and an anonymous reviewer provided valuable comments that lead us to produce a significantly more comprehensive paper than originally submitted. Efficient support for data processing was provided by Joel Doranden and Philippe Sicard. This work is part of the TOPEX/POSEIDON calibration/validation activities supported by the Centre National d'Etudes Spatiales (CNES) under contract 854/CNES/92/1709/00.

\section{References}

AVISO, AVISO User Handbook: Merged TOPEX-POSEIDON products, Rep. AVI-NT-02-10I-CN, 2nd ed., 208 pp., CNES, Toulouse, France, 1993.

Barrick, D. E., and B. J. Lipa, Analysis and interpretation of altimeter sea echo, Adv. Geophys., 27, 61-99, 1985.

Born, G. H., M. A. Richards, and G. W. Rosborough, An empirical determination of the effects of sea state bias on Seasat altimetry, J. Geophys. Res., 87, 3221-3226, 1982.

Branger, H., A. Ramamonjarisoa, and L. Bliven, A Ku-band laboratory experiment on the electromagnetic bias, IEEE Trans. Geosci. Remote Sens., 31, 1165-1179, 1993.

Callahan, P. S., TOPEX-POSEIDON Project GDR User's Handbook, Rep. D-8944, Jet Propuls. Lab., Pasadena, Calif., 1992.

Cartwright, D. E., and R. D. Ray, Oceanic tides from Geosat altimetry, J. Geophys. Res., 95, 3069-3090, 1990.

Chelton, D. B., and M. G. Schlax, Spectral characteristics of time-dependent orbit errors in altimeter height measurements, $J$. Geophys. Res., 98, 12,579-12,600, 1993.

Chelton, D. B., M. G. Schlax, D. L. Witter, and J. G. Richman, Geosat altimeter observations of the surface circulation of the Southern Ocean, J. Geophys. Res., 95, 17,877-17,903, 1990.

Douglas, B. C., and R. W. Agreen, The sea state correction for GEOS 3 and Seasat satellite altimeter data, J. Geophys. Res., 88, 1655-1661, 1983.

Efron, B., Bootstrap methods: Another look at the jacknife, Ann. Stat., 7, 1-26, 1979.

Engelis, T., Radial orbit error reduction and sea surface topography determination using satellite altimetry, Rep. 377, 178 pp., Dep. of Geod. Sci. and Surv., Ohio State Univ., Columbus, 1987.

Fu, L.-L., Report of the sea-state bias session, Minutes of the Third TOPEX/POSEIDON Science Working Team Meeting, Rep. D-8085, Jet Propuls. Lab., Pasadena, Calif., 1990.

Fu, L.-L., and R. Glazman, The effect of the degree of wave development on the sea state bias in radar altimetry measurements, J. Geophys. Res., 96, 829-834, 1991.

Fu, L.-L., and G. Pihos, Determining the response of sea level to atmospheric pressure forcing using TOPEX/POSEIDON data, $J$. Geophys. Res., this issue.

Gaspar, P., P. Y. Le Traon, J. Stum, and H. Makhmara, Determining the Geosat sea state bias from crossover data: Sensitivity studies, Eos Trans. AGU, 73(43), Fall Meeting suppl., 125, 1992.

Glazman, R. E., and A. Greysukh, Satellite altimeter measurements of surface winds, J. Geophys. Res., 98, 2475-2483, 1993. (Correction, J. Geophys. Res., 98, 14,751, 1993.)

Glazman, R. E., and S. H. Pilorz, Effects of sea maturity on satellite altimeter measurements, J. Geophys. Res., 95, 2857-2870, 1990.

Glazman, R. E., and M. A. Srokosz, Equilibrium wave spectrum and sea state bias in satellite altimetry, J. Phys. Oceanogr., 21 , I609-1621, 1991.

Glazman, R. E., A. Greysukh, and V. Zlotnicki, Evaluating models of sea state bias in satellite altimetry, J. Geophys. Res., 99, $12,581-12,591,1994$.

Haines, B. J., G. H. Born, G. W. Rosborough, J. G. Marsh, and R. G. Williamson, Precise orbit computations for the Geosat Exact Repeat Mission, J. Geophys. Res., 95, 2871-2886, 1990.

Hasselmann, D. E., M. Dunckel, and J. A. Ewing, Directional wave spectra observed during JONSWAP 1973, J. Phys. Oceanogr., 10, 1264-1280, 1980.

Hayne, G. S., and D. W. Hancock, Sea state-related altitude errors in the Seasat altimeter, J. Geophys. Res., 87, 3227-323I, 1982.
Hevizi, L. G., E. J. Walsh, R. E. Mclntosh, D. Vandemark, D. E. Hines, R. N. Swift, and J. F. Scott, Electromanetic bias in sea surface range measurements at frequencies of the TOPEX/ POSEIDON satellite, IEEE Trans. Geosci. Remote Sens., 31 , 376-388, 1993.

Jackson, F. C., The reffection of impulses from a nonlinear random sea, J. Geoply's. Res., 84, 4939-4943, 1979.

Le Traon, P. Y., J. Stum, J. Dorandeu, P. Gaspar, and P. Vincent, Global statistical analysis of TOPEX/POSEIDON data, $J$. Geophys. Res., this issue.

Liebelt, P. B., An Introduction to Optimal Estimation Theory, 273 pp., Addison-Wesley, Reading, Mass., 1967.

Longuet-Higgins, M. S., The effect of nonlinearities on statistical distributions in the theory of sea waves, J. Fluid Mech., 17, 459-480, 1963.

Melville, W. K., R. H. Stewart, W. C. Keller, J. A. Kong, D. V. Arnold, A. T. Jessup, M. R. Loewen, and A. M. Slinn, Measurements of electromagnetic bias in radar altimetry, $J$. Geophys. Res., 96, 4915-4924, 1991.

Minster, J. F., D. Jourdan, C. Boissier, and P. Midol-Monnet, Estimation of the sea-state bias in radar altimeter Geosat data from examination of frontal systems, J. Atmos. Oceanic Technol., 9, 174-187, 1992 .

Nouel, F., et al., Precise Centre National d'Etudes Spatiales orbits for TOPEX-POSEIDON: Is reaching $2 \mathrm{~cm}$ still a challenge?, $J$. Geophys. Res., this issue.

Phillips, O. M., The Dynamics of the Upper Ocean, $336 \mathrm{pp}$, Cambridge University Press, New York, 1977.

Ray, R. D., and C. J. Koblinsky, On the sea-state bias of the Geosat altimeter, J. Atmos. Oceanic Technol., 8, 397-408, 1991.

Rodriguez, E., Altimetry for non-Gaussian oceans: Height biases and estimation of parameters, J. Geophys. Res., 93, 14,107$14,120,1988$.

Rodriguez, E., and B. Chapman, Extracting ocean surface information from altimeter returns: The deconvolution method, $J$, Geophys. Res., 94, 9761-9778, 1989.

Rodriguez, E., Y. Kim, and J. M. Martin, The effect of small-wave modulation on the electromagnetic bias, J. Geophys. Res., 97, 2379-2389, 1992.

Schrama, E. J. O., The Role of Orbit Errors in Processing of Altimeter Data, Publ, on Geod-New Ser., vol. 33, 167 pp., Netherlands Geodetic Commission, Delft, 1989.

Srokosz, M. A., On the joint distribution of surface elevation and slopes for a nonlinear random sea, with an application to radar altimetry, J. Geophys. Res., 91, 995-1006, 1986.

Stewart, R., L.-L. Fu, and M. Lefebvre, Science opportunities from the TOPEX-POSEIDON mission, $J P L$ Publ., 86-18, 58 pp., 1986.

Tai, C.-K., and L.-L. Fu, On crossover adjustments in satellite altimetry and its oceanographic implications, J. Geophys. Res., 91, 2549-2554, 1986.

Walsh, E. J., F. C. Jackson, E. A. Uliana, and R. N. Swift, Observations on electromagnetic bias in radar altimeter sea surface measurements, J. Geophys. Res., 94, 14,575-14,584, 1989.

Walsh, E. J., et al., Frequency dependence of electromagnetic bias in radar altimeter sea surface range measurements, $J$. Geophys. Res., 96, 20,571-20,583, 1991

Witter, D. L., and D. B. Chelton, A Geosat altimeter wind speed algorithm and a method for altimeter wind speed algorithm development, J. Geophys. Res., 96, 8853-8860, 1991a.

Witter, D. L., and D. B. Chelton, An apparent wave height dependence in the sea state bias in Geosat altimeter range measurements, J. Geophys. Res., 96, 8861-8867, $1991 \mathrm{~b}$.

Zanife, O.-Z., POSEIDON geophysical algorithms specifications, CLS Tech. Rep. TP-ST-6136-220, CLS, Toulouse, France, 1992. Zlotnicki, V., L.-L. Fu, and W. Patzert, Seasonal variability in global sea level observed with Geosat altimetry, J. Geophys. Res., 94, 17,959-17,969, 1989.

P. Gaspar, P.-Y. Le Traon, F. Ogor, and O.-Z. Zanife, CLS Space Oceanography Group, 4 avenue de l'Europe, 31526 Ramonville Cedex, France.

(Received December 2, 1993; revised June 3, 1994; accepted June 3, 1994.) 